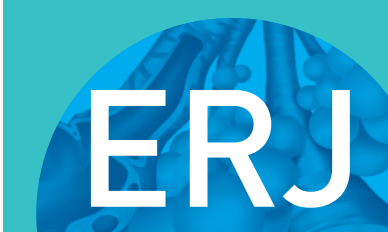

open research

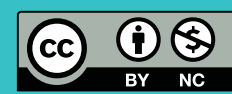

\title{
Inhaled beclomethasone/formoterol in idiopathic pulmonary fibrosis: a randomised controlled exploratory study
}

To the Editor:

We hypothesise that inflammation plays a role in idiopathic pulmonary fibrosis (IPF) and that the harm associated with corticosteroid-containing regimens in IPF may relate to local beneficial effects being counterbalanced by deleterious systemic effects $[1,2]$. Topical lung delivery could improve the poise between risk and benefit.

Inhaled corticosteroids (ICS) are widely used in airway diseases to target inflammation, often in combination with long-acting $\beta_{2}$-adrenoceptor agonists (LABA). Human lung fibroblasts express $\beta_{2}$-adrenoceptors, and agonist-induced downregulation of collagen synthesis and myofibroblast differentiation has been demonstrated in vitro [3]. Inhibition of profibrotic mediator release in response to transforming growth factor- $\beta 1$ has also been demonstrated [4]. Thus, there is a pharmacological rationale to study ICS/LABA combinations in IPF.

Platelets provide a potential link between inflammation and fibrosis, and have been shown to accumulate and correlate with collagen deposition in the lungs of animals with bleomycin induced lung injury [5]. We have demonstrated increased platelet-monocyte interactions [6] and propensity for platelet activation in response to physiological agonists in IPF patients [7]. A physiological consequence of platelet activation is thrombosis, and the association between IPF and thrombotic vascular diseases is well documented [8]. We propose that markers of platelet activation reflect a pathological process in IPF and may have utility as a biomarker.

We report the findings of an exploratory, double-blind, placebo-controlled, randomised, crossover trial of "ultrafine" inhaled beclomethasone/formoterol in IPF. Patients attending a single tertiary centre were randomised to receive either 4 weeks' treatment with a beclomethasone/formoterol (Fostair; Chiesi, Parma, Italy) $100 / 6 \mu \mathrm{g}$ hydrofluoroalkane pressurised metered-dose inhaler, two puffs twice per day, followed by matched placebo or vice versa in a $2 \times 2$ crossover design with a 4 -week washout period. Patients were eligible for inclusion if they were aged 40-85 years, had an IPF diagnosis made by a multidisciplinary team in accordance with international consensus criteria and had a forced vital capacity (FVC) of 50-110\% predicted, carbon monoxide transfer factor $\geqslant 30 \%$ predicted and oxygen saturations $\geqslant 89 \%$ while breathing room air. Potential participants were excluded if they had a secondary cause for their pulmonary fibrosis, were current smokers and/or they had used any of the following medications in the past 3 months: ICS, LABA, pirfenidone, oral corticosteroids or antiplatelet therapy that may have altered assessment of study end-points (e.g. clopidogrel, prasugrel and dipyridamole). The study was approved by the regional research ethics committee (Local Research Ethics Coordinator reference 14/YH/0053).

We chose change in platelet activation from baseline as the primary outcome of this exploratory study. Platelet-monocyte aggregate (PMA) formation, P-selectin expression and fibrinogen binding were evaluated using whole-blood flow cytometry when unstimulated and following stimulation with ADP [7].

Secondary outcome measures recorded at baseline and the end of each treatment period included lung function (forced expiratory volume in $1 \mathrm{~s}(\mathrm{FEV} 1), \mathrm{FVC}$ and forced expiratory fraction at 25-75\% of FVC (FEF25-75\%)), quality of life (the Kings Brief Interstitial Lung Disease (K-BILD) questionnaire), exercise

@ERSpublications

IPF biomarker improved by ICS/LABA http://ow.ly/CHub30gzHry

Cite this article as: Wright CE, Fraser SD, Brindle $\mathrm{K}$, et al. Inhaled beclomethasone/formoterol in idiopathic pulmonary fibrosis: a randomised controlled exploratory study. ERJ Open Res 2017; 3: 00100-2017 [https://doi.org/10.1183/23120541.00100-2017]. 
capacity (6-min walk distance (6MWD)), physical activity (mean step count over 7 days) and airway inflammation (exhaled nitric oxide fraction ( $\mathrm{FeNO}$ ) and sputum differential cell count).

Data are presented as mean $\pm \mathrm{SD}$. Differences were assessed between groups and before and after treatment using Student's t-tests. A two-tailed $\mathrm{p}<0.05$ was considered significant. Platelet activation markers were compared using the mean area under the concentration-response curve (AUC). Changes in AUC were compared using ANOVA and Tukey's post hoc test.

20 patients were screened, with 17 proceeding to randomisation and completing the study (males/females 11/6; age $71.1 \pm 8.7$ years; 10 ex-smokers, six never-smokers and one with unknown smoking history; baseline FEV1 $2.10 \pm 0.60 \mathrm{~L}(89.9 \pm 15.3 \%$ predicted), FVC $2.64 \pm 0.79 \mathrm{~L}(86.2 \pm 17.4 \%$ predicted), FEV1/FVC

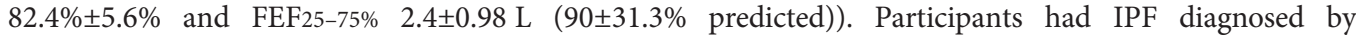
multidisciplinary assessment according to international criteria and no participants had evidence of airflow obstruction at study entry.

Beclomethasone/formoterol significantly reduced platelet P-selectin expression from baseline (AUC: baseline, 283.2 \pm 123.8 ; beclomethasone/formoterol, 196.9 $\pm 72 ; \mathrm{p}<0.05)$. PMA formation and fibrinogen binding showed trends to reduction compared to baseline. Compared to placebo, there were trends towards reduced platelet reactivity (P-selectin: AUC 196.9 \pm 72 following beclomethasone/formoterol and 224.3 \pm 79.9 following placebo; PMA: $330.2 \pm 180.6$ and 381.8 \pm 224.3 ; fibrinogen: $685.7 \pm 188.3$ and $769.1 \pm 154.5$, respectively). The greatest differences were observed following stimulation with higher concentrations of ADP (figure 1).

Beclomethasone/formoterol improved FEV1 (+0.07 L versus placebo, $\mathrm{p}<0.05)$ and $\mathrm{FEF} 25-75 \%(+0.19 \mathrm{~L}$ versus placebo, $\mathrm{p}<0.05$ ) (figure 1). Sputum eosinophil counts decreased from $5.7 \pm 5.6 \%$ at baseline to $2.2 \pm 2.5 \%$ following placebo (nonsignificant) and $1.2 \pm 1.2 \%$ following beclomethasone/formoterol $(\mathrm{p}<0.05$ compared to baseline, nonsignificant compared to placebo). No changes were observed in FVC, K-BILD, 6MWD, mean step count or FeNO.

The findings of this exploratory study demonstrate the potential for this inhaled combination therapy to tackle various aspects of this complex and poorly understood condition. ICS therapy targets the inflammatory process whereas the LABA contributes to bronchodilation and has activity on platelet activation.
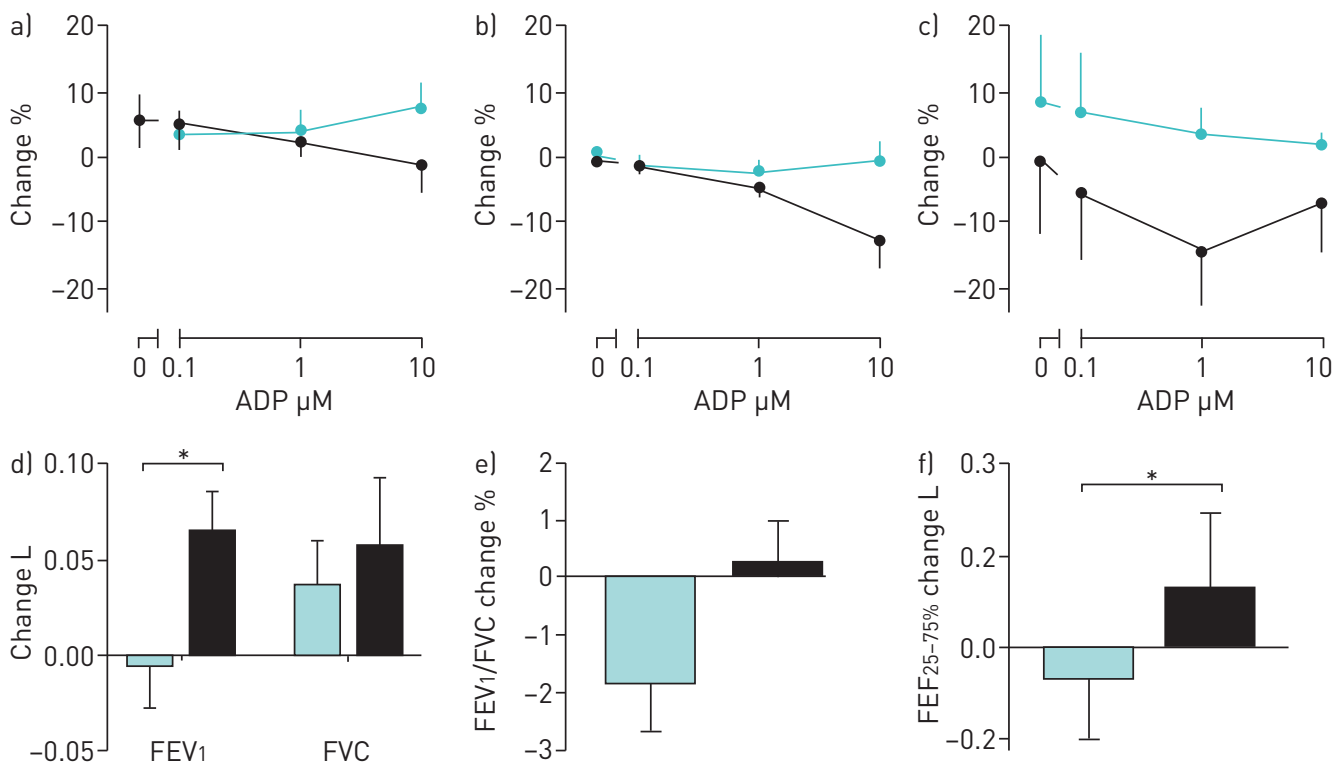

- Beclometasone/formoterol $\longrightarrow \square$ Placebo

FIGURE 1 Change from baseline following beclomethasone/formoterol and placebo in al platelet-monocyte aggregate formation, b) P-selectin expression and c) platelet fibrinogen expression when unstimulated and following stimulation with ADP $0.1-10 \mu \mathrm{M}$. Change from baseline in d) forced expiratory volume in $1 \mathrm{~s}$ (FEV 1 ] and forced vital capacity (FVC), e) forced expiratory ratio and f) forced expiratory fraction at $25-75 \%$ of FVC (FEF25-75\%) following treatment with beclomethasone/formoterol versus placebo. Data are presented a mean \pm SEM. $*$ : $p<0.05$ 
Platelet activation leads to proinflammatory and profibrotic mediator release. Therefore, the increased platelet reactivity observed in IPF patients has potential pathophysiological consequences. Despite higher than expected variability of the platelet assays and our small sample size limiting our ability to demonstrate statistically significant change, consistent trends towards reduced platelet reactivity were observed across the range of markers in response to treatment. Beclomethasone/formoterol may affect platelet reactivity directly through activity on platelet $\beta_{2}$-adrenoceptors [9] and potentially glucocorticoid receptors [10] (the presence of glucocorticoid receptors on platelets remains contentious), or indirectly though reduction of inflammation and fibrosis.

By using inhaled beclomethasone/formoterol with extra-fine particles $(1.5 \mu \mathrm{m})$, we aimed to achieve delivery of the drug to peripheral airways [11] where abnormalities have been demonstrated in IPF lung explants [12]. It is interesting that we observed improvements in FEV1 and FEF25-75\% from baseline compared to placebo, with the latter measure often considered to reflect small airways. This supports the presence of a potentially modifiable pathology in the small airways of these patients who, at study entry, had no physiological evidence of airflow obstruction.

The sputum eosinophil data support previous demonstration of sputum eosinophilia in IPF patients [13]. The observed reduction in sputum eosinophilia during the study was most marked following beclomethasone/formoterol but also occurred, to a lesser extent, following placebo. The small proportion of patients that were able to provide sputum samples at every study time-point limits the generalisability of this finding but it supports the hypothesis that there is modifiable inflammation in IPF airways.

We excluded patients with demonstrable airflow obstruction and no participants in this study had obstructive spirometry $(\mathrm{FEV} 1 / \mathrm{FVC}$ ratio $<0.7)$. Although we cannot rule out nascent obstructive airway disease, we believe our patients are typical of those presenting to specialist interstitial lung disease clinics and suggest that the small airways may represent a neglected therapeutic target in IPF.

\section{Caroline E. Wright, Simon D. Fraser, Kayleigh Brindle, Alyn H. Morice, Simon P. Hart and Michael G. Crooks}

Centre for Cardiovascular and Metabolic Research, Hull York Medical School, Hull, UK.

Correspondence: Michael G. Crooks, Centre for Cardiovascular and Metabolic Research, Hull York Medical School, Castle Hill Hospital, Cottingham, HU16 5JQ, UK. E-mail: michael.crooks@nhs.net

Received: Aug 142017 | Accepted after revision: Oct 202017

This trial is registered at eudract.ema.europa.eu with identifer number 2013-004404-19

Support statement: This research was funded by Chiesi Ltd. Funding information for this article has been deposited with the Crossref Funder Registry.

Conflicts of interest: Disclosures can be found alongside this article at openres.ersjournals.com

Acknowledgements: A.H. Morice, S.P. Hart and M.G. Crooks conceived and designed the study; K. Brindle, C.E. Wright and S.D. Fraser acquired the data during the study, and S.D. Fraser, C.E. Wright, A.H. Morice, S.P. Hart, M.G. Crooks analysed and interpreted those data; C.E. Wright, A.H. Morice, S.P. Hart and M.G. Crooks drafted the manuscript, and all authors critically reviewed it and approved the submitted version.

\section{References}

$1 \quad$ Piguet PF. Inflammation in idiopathic pulmonary fibrosis. Am J Respir Crit Care Med 2003; 167: 1037.

2 Roghair RD, Volk KA, Lamb FS, et al. Impact of maternal dexamethasone on coronary $\mathrm{PGE}_{2}$ production and prostaglandin-dependent coronary reactivity. Am J Physiol Regul Integr Comp Physiol 2012; 303: R513-R519.

3 Lamyel F, Warnken-Uhlich M, Seeman WK, et al. The $\beta 2$-subtype of adrenoceptors mediates inhibition of pro-fibrotic events in human lung fibroblasts. Naunyn Schmiedebergs Arch Pharmacol 2011; 384: 133-145.

4 Tannheimer SL, Wright CD, Salmon M. Combination of roflumilast with a beta-2 adrenergic receptor agonist inhibits proinflammatory and profibrotic mediator release from human lung fibroblasts. Respir Res 2012; 13: 28.

5 Piguet PF, Vesin C. Pulmonary platelet trapping induced by bleomycin: correlation with fibrosis and involvement of the beta 2 integrins. Int J Exp Pathol 1994; 75: 321-328.

6 Fahim A, Crooks MG, Morice AH, et al. Increased platelet binding to circulating monocytes in idiopathic pulmonary fibrosis. Lung 2014; 192: 277-284.

7 Crooks MG, Fahim A, Naseem KM, et al. Increased platelet reactivity in idiopathic pulmonary fibrosis is mediated by a plasma factor. PLoS One 2014; 9: e111347.

8 Hubbard RB, Smith C, Le Jeune I, et al. The association between idiopathic pulmonary fibrosis and vascular disease: a population-based study. Am J Respir Crit Care Med 2008; 178: 1257-1261.

9 Larsson PT, Wallen NH, Martinsson A, et al. Significance of platelet beta-adrenoceptors for platelet responses in vivo and in vitro. Thromb Haemost 1992; 68: 687-693. 
10 Moraes LA, Paul-Clark MJ, Rickman A, et al. Ligand-specific glucorticoid receptor activiation in human platelets. Blood 2005; 106: 4167-4175.

11 Wilfried DB, Annick D, Gianluigi P, et al. Lung deposition of BDP/formoterol HFA pMDI in healthy volunteers, asthmatic, and COPD patients. J Aerosol Med Pulm Drug Deliv 2010; 23: 137-148.

12 Verleden S, Piloni D, Willems S, et al. Small airway obstruction in idiopathic pulmonary fibrosis. Am J Respir Crit Care Med 2015; 191: A6325.

13 Birring SS, Parker D, Mckenna B, et al. Sputum eosinophillia in idiopathic pulmonary fibrosis. Inflamm Res 2005; 54: $51-56$. 